\title{
REESTRUTURAÇÃO DA GESTÃO DAS INFORMAÇÕES ESTRATÉGICAS DA CSNPR*
}

\author{
Janete Terezinha Ferreira da Silva Lecheta ${ }^{1}$ \\ Rafael Carvalho Lara ${ }^{2}$ \\ Robson Serafim Gimenes ${ }^{3}$
}

\section{Resumo}

A CSN Paraná, sempre trabalhando com a cultura de melhoria contínua, passou recentemente por uma reestruturação na gestão das informações estratégicas, mantendo o que já existia de eficiente e aplicando melhorias e novidades que trouxeram ganhos de produtividade, suporte na tomada de decisão e outros ganhos intangíveis. O breve estudo tem como objetivo apresentar o trabalho que foi feito na CSN Paraná, os conceitos utilizados e metodologias aplicadas para que esse trabalho fosse possível.

Palavras-chave: Sistema de gestão; Indicadores de desempenho; Estratégia.

\section{MANAGEMENT OF RESTRUCTURING OF STRATEGIC INFORMATION CSN PARANÁ}

\section{Abstract}

The CSN Paraná, always working with the culture of continuous improvement, has recently undergone a restructuring in the management of strategic information, keeping what was already efficient and implement improvements and new developments that brought productivity gains, support decision-making and other intangible gains. The brief study aims to present the work that was done on CSN Paraná, concepts and methodologies used to make this work possible.

Keywords: Management systems; Performance indicator; Strategy.

1 Análise de Sistemas, Bacharel em Análise de Sistemas, Analista de Negócios, Gerência de Sistemas de Informática, Companhia Siderúrgica Nacional, Araucária, Paraná, Brasil.

2 Engenharia Mecânica, Engenheiro Mecânico, Especialista em Garantia da Qualidade, Gerência da Garantia da Qualidade, Companhia Siderúrgica Nacional, Araucária, Paraná, Brasil.

3 Sistemas de Informação, Bacharel em Sistemas de Informação, Analista de Desempenho Operacional, Companhia Siderúrgica Nacional, Araucária, Paraná, Brasil. 


\section{INTRODUÇÃO}

A gestão estratégica é hoje, um dos principais desafios nas organizações. O cenário dos negócios está cada vez mais imprevisível, dinâmico e competitivo, reforçando a necessidade de gerenciar a estratégia de forma contínua. A estratégia necessita ser gerenciada, entretanto, gerenciar é uma atividade complexa e requer mudança cultural e de atitude em todos os níveis da organização.

A gestão estratégica consiste em fazer com que a estratégia definida seja sistematicamente acompanhada, analisada e, se for preciso, redefinida, para garantir que a visão de futuro, objetivo maior da organização, seja alcançada.

A alta direção carece de mecanismos efetivos de acompanhamento da estratégia e suporte à tomada de decisões, fundamentados em indicadores que reflitam 0 progresso da organização em direção à sua visão de futuro. Os gestores são os responsáveis pela formulação da estratégia, porém, é imprescindível o desdobramento dela até o nível operacional da organização. A estratégia é executada pelas equipes dentro dos processos que estão inseridas. As pessoas precisam conhecer o rumo que a organização está tomando para poder executar, melhorar e rever seus processos de forma alinhada com a estratégia.

A CSN Paraná deu um grande salto em termos de gestão. Estruturou-se durante os últimos anos para modelar e implantar a sistemática de gestão da estratégia culminando em 2012 na implantação de solução sistêmica (Cognos e SESuite) para sustentação, conexão e robustez do modelo

\section{MATERIAIS E MÉTODOS}

\subsection{Sistema de Liderança da CSN-PR}

O sistema de gestão da CSN Paraná tem início na definição da Visão, Missão, Política, Objetivos e Valores pela direção. Em seguida, o gerente-geral junto aos gerentes e coordenadores, utiliza o conceito de planejamento estratégico para formular as estratégias e desenhar o Mapa Estratégico, o qual é o direcionador dos objetivos estratégicos, ou seja, é a base para o desdobramento dos objetivos e metas em indicadores de medição dos processos.

$\mathrm{Na}$ etapa seguinte são construídos os mapas de processos, onde se estabelece as entradas e saídas, os métodos, os recursos, os fornecedores e os indicadores de desempenho para cada processo da CSN Paraná. Os indicadores de desempenho são classificados em dois tipos: indicadores de esforço e indicadores de resultado, conforme referência da Fundação Nacional da Qualidade (FNQ).

Os indicadores dos processos são denominados como indicadores de esforço, pois eles são os responsáveis em medir os esforços dos empregados no atingimento das metas, que por consequência, contribuirão no atingimento dos indicadores dos objetivos estratégicos, os quais são denominados como indicadores de resultado.

Periodicamente são realizadas Reuniões de Análise Crítica da Direção, onde são verificados os resultados dos indicadores e o atingimento da Visão. 


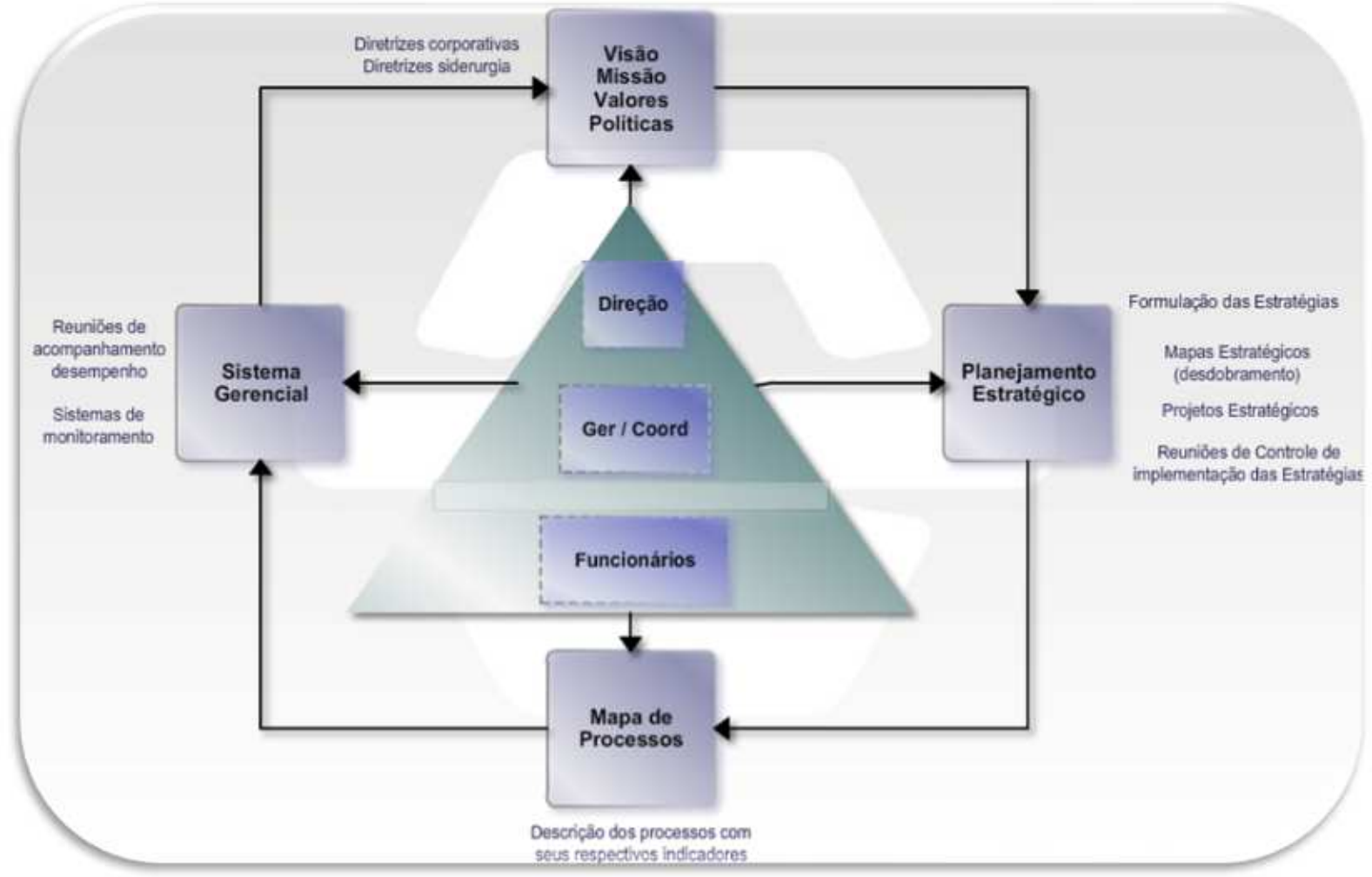

Figura 1 - Sistema de Liderança

\subsection{Sistema de Gestão da CSN-PR}

O modelo de sistema de gestão da CSN Paraná é estruturado em três grandes blocos: gestão estratégica baseada no BSC, gestão da rotina e gestão de melhorias. Estes três blocos são suportados pelas certificações: ISO 9001, ISO TS 16949, ISO 14001 e Diretiva ROHS.

No bloco gestão da estratégia é onde se realiza o mapa estratégico e o desdobramento dos objetivos, indicadores e metas.

Através das saídas da gestão estratégica é arquitetada a gestão da rotina, a qual é fortemente estruturada no processo de qualificação de mão de obra (PQMO), que é baseado em padrões, treinamento e cumprimento dos padrões. Na gestão da rotina também são analisados os resultados dos indicadores de monitoramento dos processos. Para os casos onde a meta não é atingida, é gerado como saída uma ação corretiva para o reestabelecimento da meta.

No bloco gestão de melhorias, que acontece através da reunião de análise crítica pela direção, é tratado as causas sistêmicas que não foram solucionadas na gestão da rotina. As saídas da reunião de análise crítica da direção são direcionadas em projetos de melhorias que retroalimentam o próximo ciclo do planejamento estratégico. 


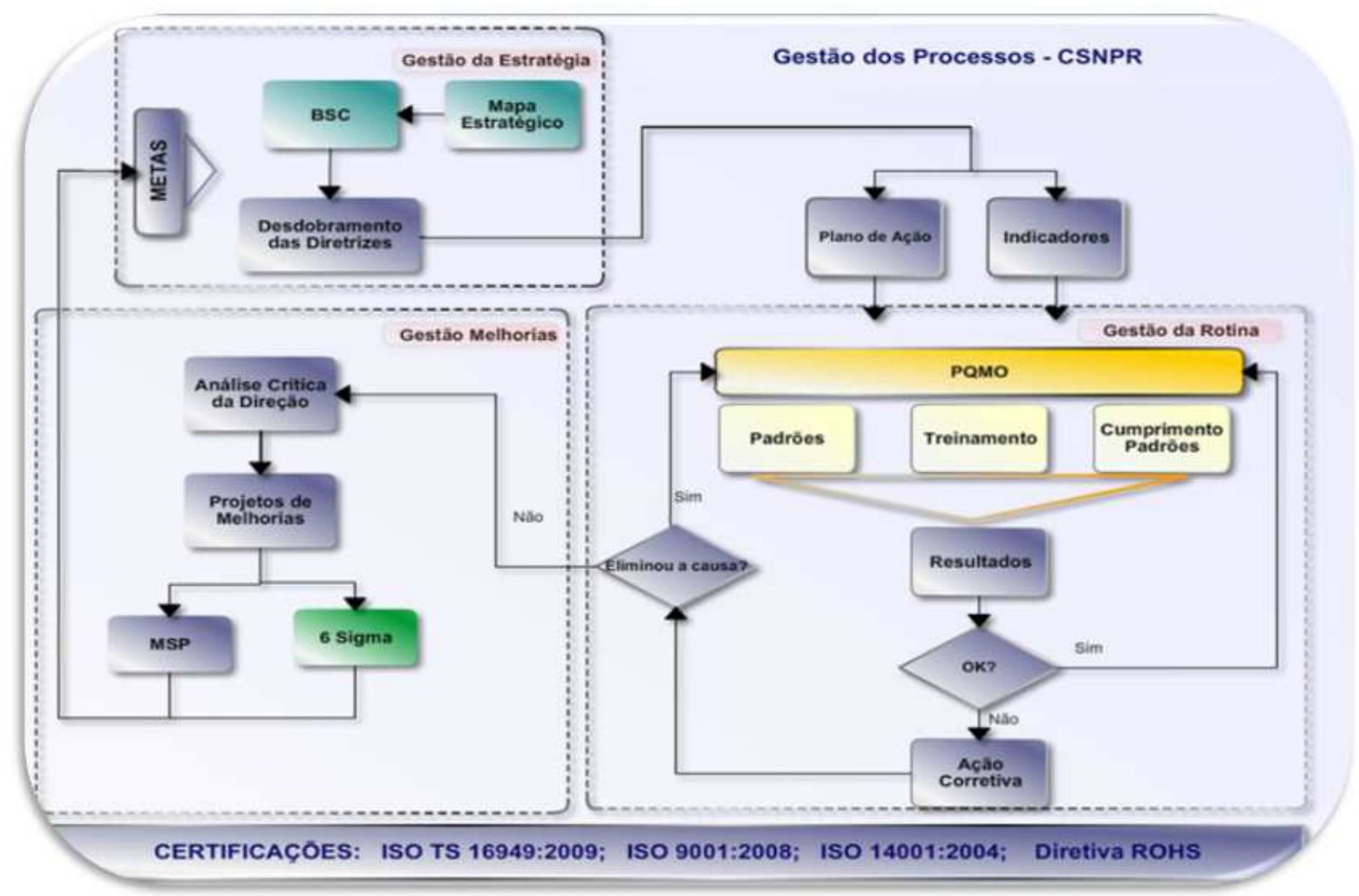

Figura 2 - Gestão dos Processos

\subsection{Mapa Estratégico}

O primeiro passo para a utilização do BSC nas organizações é o desenho do mapa estratégico. O mapa estratégico tem como finalidade decodificar os processos de escolha frente aos quais os gestores de todos os níveis estão expostos diariamente e também esclarecer e traduzir a estratégia. É mediante o mapa estratégico que a alta administração monitora o cumprimento da estratégia.

O mapa estratégico é composto pelos objetivos, metas e ações dispostos nas quatro perspectivas de gestão: financeira, de clientes, de processos internos e de aprendizado e crescimento.

O mapa estratégico fornece a representação visual para a integração dos objetivos da organização nas quatro perspectivas do BSC e ilustra as relações de causa e efeito conectando os resultados almejados para cada perspectiva. Nesse mapeamento é possível observar a relação sistêmica entre as variáveis numa relação causa/efeito. A partir dessa análise, os gestores poderão identificar os pontos a serem repensados numa perspectiva sistêmica e com a visualização imediata na própria ferramenta.

Para Kaplan e Norton [1], a perspectiva financeira descreve os resultados tangíveis da estratégia. Algumas medidas como lucratividade, rentabilidade, valor para acionistas e geração de receita, indicam se a estratégia da organização está em rumo ao sucesso ou ao fracasso. No ponto de vista do cliente, identifica-se qual a proposição de valor que atinge a necessidade do mesmo. Se o cliente preza por qualidade e pontualidade, esses valores devem ser traduzidos como fundamentais para a organização. A perspectiva que enfatiza os processos internos identifica quais processos se espera que exerçam maior impacto sobre a estratégia, e trabalha fortemente sobre eles. Por fim, a perspectiva do aprendizado e crescimento destaca-se como os ativos intangíveis de maior importância para a estratégia, ou 
seja, o capital humano, o clima organizacional e o sistema (capital da informação) são necessários para os processos de criação de valor, o qual embasa a utilização do BSC.

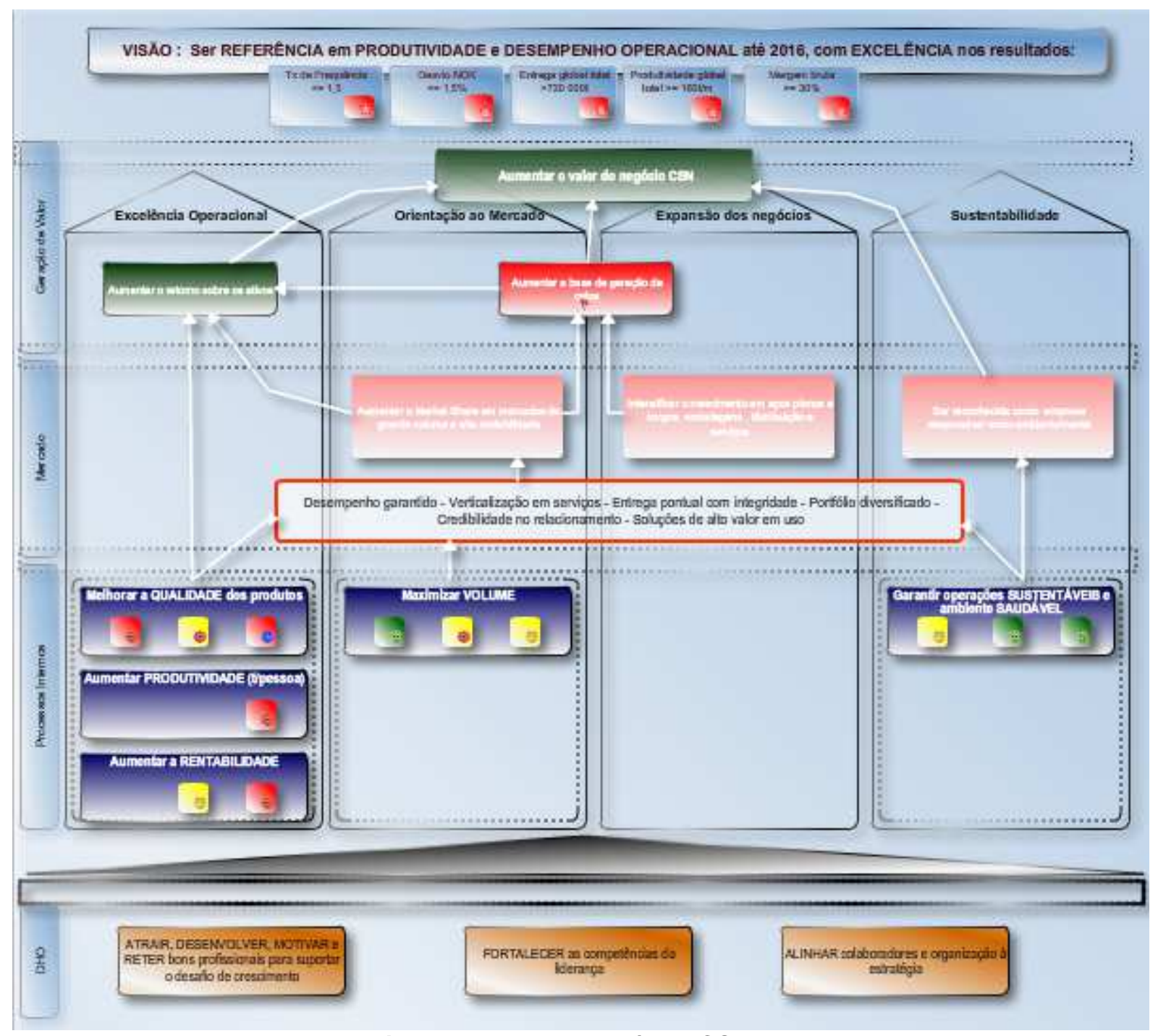

Figura 3 - Mapa Estratégico CSN-PR

\subsection{Metodologia do Trabalho}

O trabalho foi desenvolvido utilizando a metodologia de desenvolvimento de projetos da Diretoria de Informática da CSN. Esta metodologia é baseada nas nove áreas de conhecimento do PMBOK: 
Tabela 1 - áreas de conhecimento do PMBOK

\begin{tabular}{|c|c|}
\hline ÁREA CONHECIMENTO PMBOK & PROCESSOS DO GERENCIAMENTO \\
\hline Gerenciamento do Escopo & $\begin{array}{l}\text { Iniciação, } \\
\text { Planejamento do Escopo, } \\
\text { Detalhamento do Escopo, } \\
\text { Verificação do escopo, } \\
\text { Controle das Mudanças do Escopo }\end{array}$ \\
\hline Gerenciamento da Integração & $\begin{array}{l}\text { Desenvolvimento do Plano de Projeto, } \\
\text { Execução do Plano de Projeto, } \\
\text { Controle Integrado de Mudanças, } \\
\text { Desenvolvimento do Plano de Projeto, } \\
\text { Execução do Plano de Projeto, } \\
\text { Controle Integrado de Mudanças }\end{array}$ \\
\hline Gerenciamento do Tempo & $\begin{array}{l}\text { Definição das Atividades, } \\
\text { Sequenciamento das Atividades, } \\
\text { Estimativa da Duração, } \\
\text { Desenvolvimento do Cronograma, } \\
\text { Controle do Cronograma }\end{array}$ \\
\hline Gerenciamento dos Custos & $\begin{array}{l}\text { Planejamento dos Recursos, } \\
\text { Estimativa dos custos, } \\
\text { Orçamento dos custos, } \\
\text { Controle dos custos }\end{array}$ \\
\hline Gerenciamento da Qualidade & $\begin{array}{l}\text { Planejamento da Qualidade, } \\
\text { Garantia da Qualidade, } \\
\text { Controle da Qualidade }\end{array}$ \\
\hline Gerenciamento dos Recursos Humanos & $\begin{array}{l}\text { Planejamento Organizacional, } \\
\text { Montagem da Equipe, } \\
\text { Desenvolvimento da Equipe }\end{array}$ \\
\hline Gerenciamento da Comunicação & $\begin{array}{l}\text { Planejamento das Comunicações, } \\
\text { Distribuição das Informações, } \\
\text { Relato de Desempenho, } \\
\text { Encerramento Administrativo }\end{array}$ \\
\hline Gerenciamento dos Riscos & $\begin{array}{l}\text { Planejamento da Gerência de Riscos, } \\
\text { Identificação dos Riscos, } \\
\text { Análise Qualitativa dos Riscos, } \\
\text { Análise Quantitativa dos Riscos, } \\
\text { Desenvolvimento de Resposta a Riscos, } \\
\text { Controle e Monitoração de Riscos }\end{array}$ \\
\hline Gerenciamento das Aquisições & $\begin{array}{l}\text { Planejamento das Aquisições, } \\
\text { Preparação das Aquisições, } \\
\text { Obtenção de Propostas, } \\
\text { Seleção de Fornecedores, } \\
\text { Administração dos Contratos, } \\
\text { Encerramento do Contrato }\end{array}$ \\
\hline
\end{tabular}

Para que um projeto seja iniciado, conforme o Modelo de Gestão de Projetos vigente na Diretoria de Informática da CSN, é necessário que este tenha sido priorizado segundo as normas de governança e tenha seu orçamento aprovado pela Diretoria Executiva. O projeto de implantação de solução para suportar o modelo de gestão da CSNPR foi aprovado no orçamento em 2010 baseado na norma NG1303.05 POLÍTICA DE GESTÃO E PRIORIZAÇÃO DE DEMANDAS DE TI. 
Os projetos são compostos de processos distribuídos em fases. Na figura abaixo está o fluxo da metodologia de gestão de projetos:

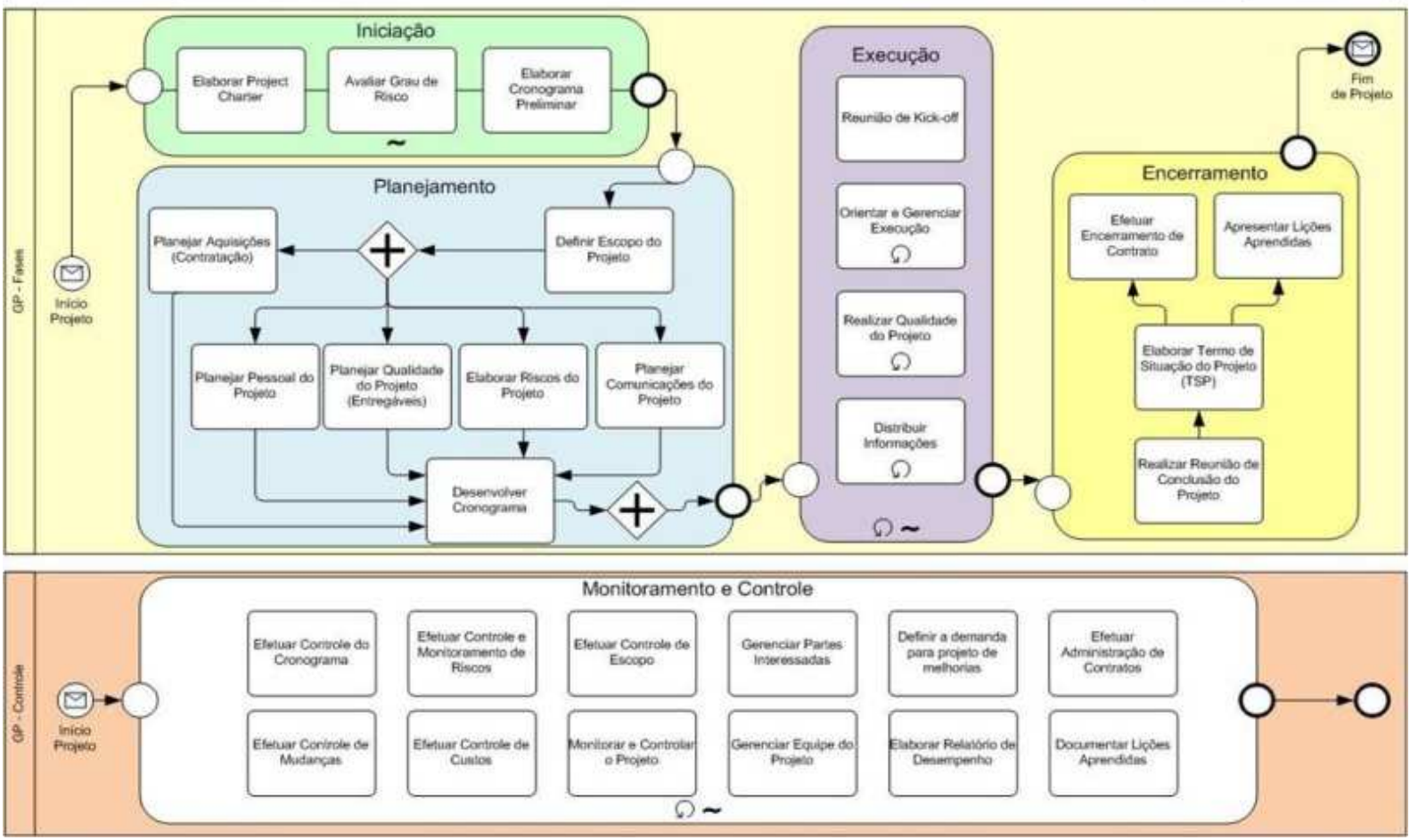

Figura 4 - Metodologia de projeto

\section{RESULTADOS E DISCUSSÃO}

\subsection{Histórico de Evolução do Sistema de Gestão CSNPR}

A CSN Paraná vem ao longo de sua história aprimorando seus processos. Em 2003, logo após a implantação do projeto da fábrica, os processos não eram mapeados, nem padronizados e muito menos monitorados através de indicadores, e isso contribuía para geração de produtos não-conformes e reclamações de clientes a níveis elevados. Além disso, o maior cliente da CSN Paraná, a Whirlpool, estava cobrando a certificação ISO 9001. A partir desse momento a direção da CSN Paraná decidiu pela implantação do sistema de gestão baseado na ISO 9001.

No ano de 2004, foi iniciado o projeto ISO 9001 e depois de dois anos de implantação a CSN Paraná recebeu a certificação. Este modelo de sistema de gestão propiciou o estabelecimento de uma estrutura de abordagem por processo, com indicadores para monitoramento destes processos e a busca pela melhoria contínua.

Em 2007, a direção da CSN Paraná consciente da necessidade da evolução do seu sistema de gestão, decidiu pela implantação do sistema de gestão ambiental ISO 14001, a qual foi motivada pela necessidade de melhorar o seu compromisso com 0 Meio Ambiente. Em 2008 a CSN Paraná foi certificada na ISO 14001.

Com uma liderança para melhoria do sistema de gestão e com uma visão de longo prazo, a direção sentiu a necessidade de fortalecer o sistema de gestão no nível estratégico. Então, em 2008 se estabeleceu a Visão/Missão, a estratégia e desenhou-se o mapa estratégico da CSN Paraná, onde os objetivos estratégicos, indicadores e metas foram desdobrados nos processos da CSN Paraná. 
Em 2009, com o desenvolvimento de chapa de tanque de combustível para atendimento ao cliente Fiat, a CSN Paraná iniciou o projeto para implantação da ISO TS 16949. Em apenas um ano depois a CSN Paraná recebeu essa certificação. É válido lembrar que sem essa certificação a empresa não poderia fornecer produtos à Fiat.

Paralelamente aos processos de certificações da CSNPR ocorreu a adoção de sistema de apoio para suportar os controles necessários na manutenção dos processos.

Em 2006 houve a implantação do sistema ISO DOC voltado para controle de documentos e a implantação do sistema ISO Training voltado para controle de treinamentos.

No ano de 2007 ocorreu a implantação do Sistema SeSuite Ações voltado para controle de ações oriundas de não conformidades.

O corpo gestor da CSNPR, motivado pelos benefícios que as certificações trouxeram aos seus processos, bem como, alinhados aos objetivos estratégicos da CSN incentivou de forma incondicional a formulação de um modelo de gestão da CSN Paraná focado na estratégia, sendo que esta desdobra até o nível operacional. O modelo foi concebido em 2009 e implantado em 2010.

Apesar de estruturado, o modelo era de difícil manutenção, pois era todo alimentado de forma manual, sem a utilização de um sistema informatizado. Os dados eram obtidos através de consulta em sistemas especialistas e na sequência realizavam-se os cálculos dos indicadores de forma manual para posterior lançamento em tabelas em Excel, as quais geravam os gráficos de acompanhamento dos indicadores estratégicos e dos processos.

A implantação da árvore de indicadores (figura 5) foi um diferencial expressivo no acompanhamento de indicadores da CSNPR, porém, a manutenção dela também era de forma manual, demandando elevado tempo na atualização e sujeita a erros.

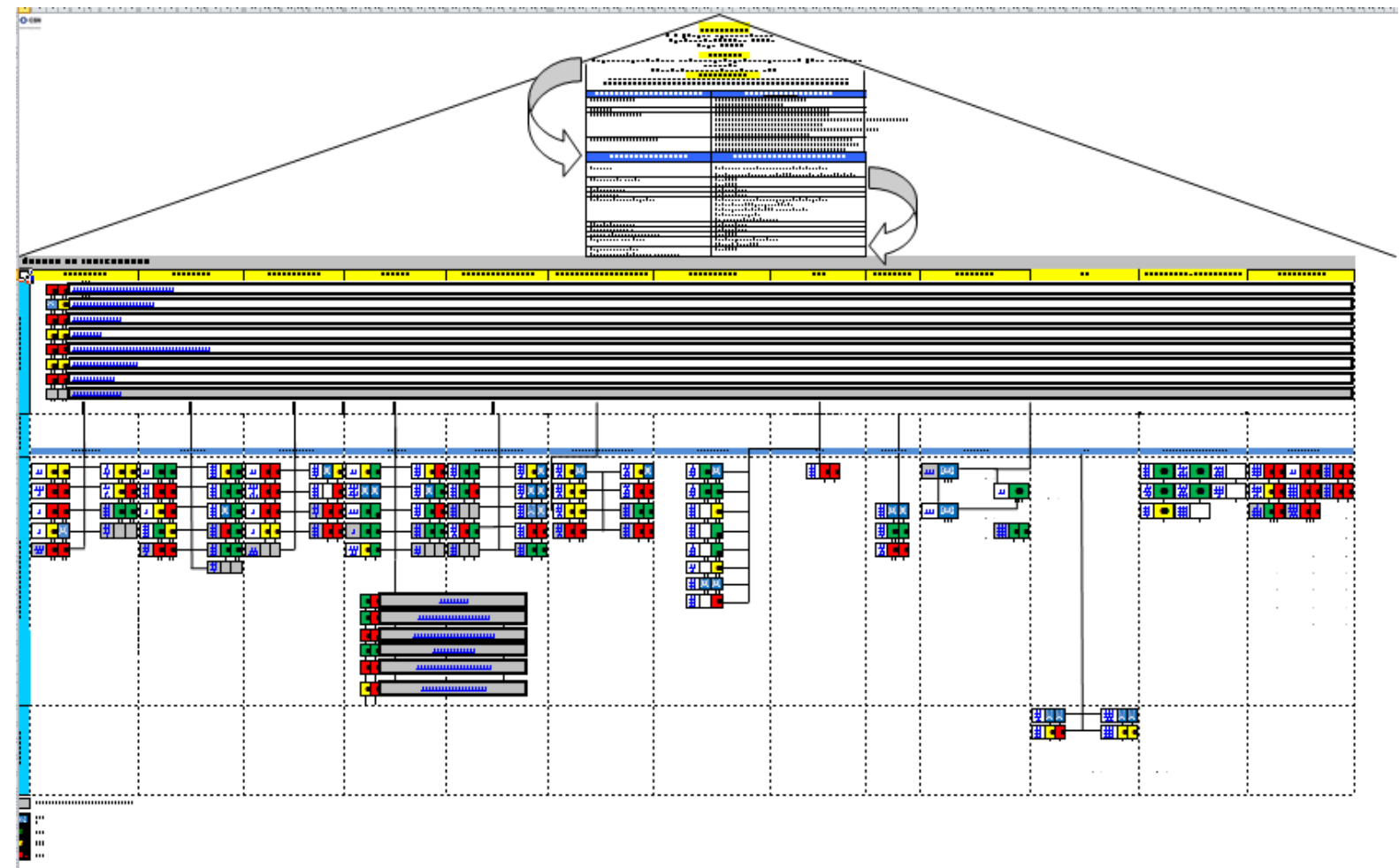

Figura 5 - Árvore de Indicadores 


\subsection{Motivadores do Projeto}

Então, nasce nesse momento o projeto de reestruturação da gestão das informações estratégicas da CSN Paraná. Nesse projeto foram gerados duas frentes de trabalho:
A. Migração do modelo de gestão da CSN Paraná para módulo SeSuite Desempenho objetivando melhorar os seguintes pontos:
- Elevado tempo para atualização dos mais de 100 indicadores;
- Abertura manual de RAC e RAP - elevado tempo na análise do critério de abertura;
- Retrabalho para garantir acuracidade dos indicadores;
- Dificuldade na análise crítica dos indicadores em reuniões gerenciais;
- Impossibilidade de realizar correlação de indicadores;
- Dependência de pessoas para obter informações;
- Vulnerabilidade dos indicadores - armazenados em planilhas;
B. Migração do Impromptu para sistema Cognos objetivando resolver:
- Descontinuidade do Impromptu pelo fabricante;
- Impossibilidade de aquisição de novas licenças;
- Divergência de informações devido a várias "bases";
- Dependência de pessoas para obter informações;
- Incompatibilidade da estrutura de informações com o sistema de gestão da CSN Paraná;

\subsection{Planejamento}

Com base nos motivadores do projeto, analisados durante a fase de Iniciação, a equipe definiu que algumas ações seriam necessárias para que o objetivo fosse alcançado, sendo elas:

- Geração de gráficos customizados conforme modelo de Gestão desenvolvido pela CSN Paraná;

- Funcionalidade para correlação de indicadores;

- Mecanismo para agilizar a entrada de dados no SeSuite;

- Abertura de RAC / RAP automática conforme regra estabelecida;

- Centralização dos requisitos / diretrizes do sistema de gestão da qualidade alinhados aos indicadores de desempenho;

- Migração dos relatórios do sistema antigo (Impromptu) para o Cognos;

- Padronização / Centralização dos relatórios gerenciais, eliminando as redundâncias e relatórios obsoletos;

- Criação de dashboards / painel com gráficos de gestão;

- Eliminar a base em Microsoft Access utilizada nos processos de produção, qualidade, estoque, atendimento a carteira;

A equipe do projeto foi composta por: Carlos Frederico Rangel Xavier (Patrocinador), Rafael Carvalho Lara, Janete Terezinha Ferreira da Silva Lecheta, Robson Serafim Gimenes, Isabel Cristina Pedroso Eduardo. A equipe também recebeu apoio de Rondinelly Moutinho Ramos (CSN), Thiago De Andrade Silva (CSN), Paulo Henrique Tonon (CSN), da DTIN de um modo geral e dos parceiros da Soft Expert e do Grupo $\mathrm{GCl}$. 
Segue abaixo o cronograma dos projetos de implantação das ferramentas SeSuite e Cognos:

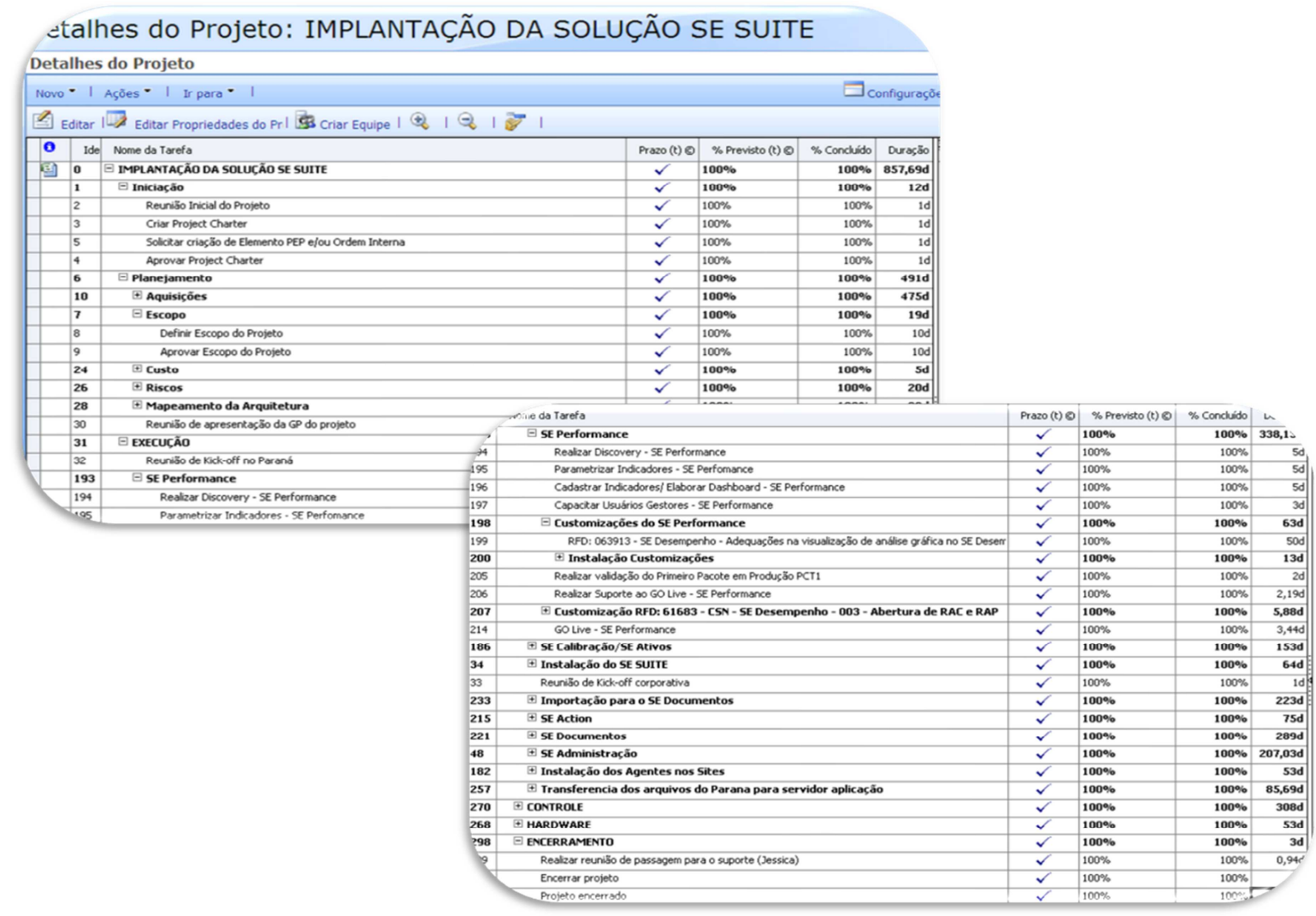

Figura 6 - Cronograma de Implantação SeSuite

etalhes do Projeto: Migração Impromptu CSN PR
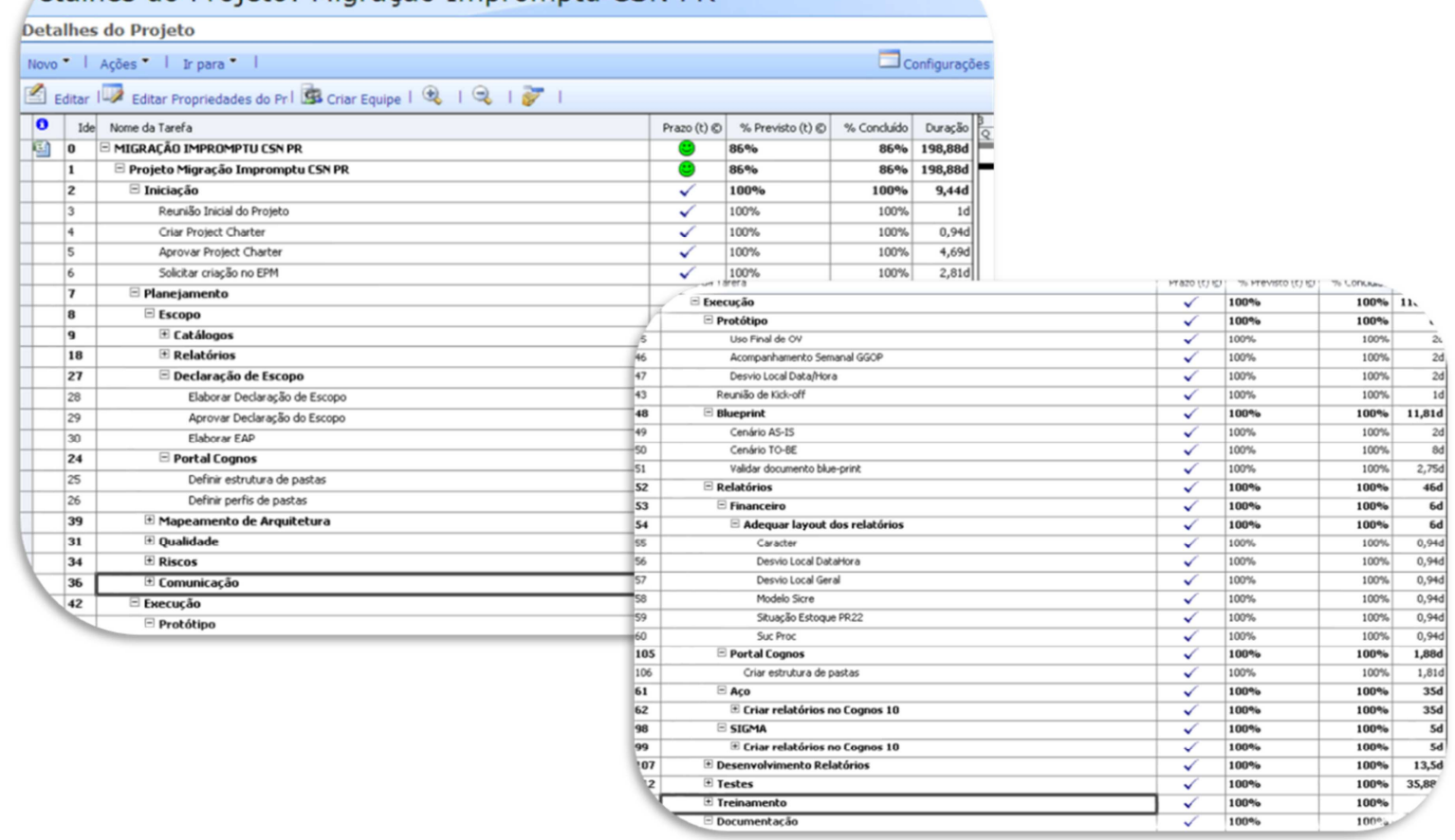

Figura 7 - Cronograma de Implantação Cognos (migração Impromptu) 


\subsection{Execução}

Como dito anteriormente a CSN Paraná possuía uma sistemática de gestão estabelecida, porém sem tecnologia da informação envolvida, tornando o processo demorado e falho (por ser um processo mantido manualmente). Consequentemente a produtividade da equipe era afetada e nem sempre era possível à direção uma tomada de decisão rápida e eficaz devido à demora na disponibilização das informações. Com a implantação dos sistemas Cognos e SeSuite, foi possível transcrever o que já existia para dentro de um sistema informatizado, criando assim um Modelo de Gestão.

O Cognos é uma ferramenta que possibilita a criação de relatórios contendo dados oriundos de Sistemas Especialistas. Esses dados podem ser, por exemplo: dados de produção, desvios, faturamento, manutenção e estoque de produtos.

O SeSuite é uma ferramenta voltada para o suporte, manutenção e atendimento das demandas críticas para a excelência no desempenho organizacional em um único ambiente integrado.

No momento da migração do sistema antigo para o Cognos, foram revisados os relatórios existentes na CSN Paraná e as seguintes ações foram tomadas:

- Eliminar relatórios redundantes ou desnecessários - havia mais de uma equipe fazendo um mesmo relatório e outros que eram atualizados, porém não mais utilizados. Também foi centralizada a geração desses relatórios gerenciais em uma única área;

- Revisar regras e conceitos dos relatórios - como havia redundância de relatórios, foi identificado que o conceito sobre um determinado assunto não era universal;

- Classificar os relatórios por processos - assim como no modelo utilizado para indicadores, optou-se por separar os relatórios (já revisados e consolidados) dentro do Cognos abordando-os em processos (Produção, Faturamento, Qualidade, Manutenção, PCP);

Na configuração do Módulo de Desempenho do SeSuite, foi levado para dentro do sistema a sistemática de gestão que já existia junto com algumas melhorias, desta forma o Modelo de Gestão Informatizado já "nasceu" nos padrões e modelos de estabelecidos pela CSN Paraná. A seguinte estrutura foi montada:

- Estruturação do Sistema de Gestão - contém breve histórico sobre a unidade da CSN-PR, fluxo de produção, sistema de liderança, visão, missão, valores, política, princípios da gestão DEPRO, gestão dos processos, gestão da estratégia, mapa estratégico, projetos estratégicos, mas dos processos e estrutura organizacional - através desse tópico pode ser feito uma apresentação do que é e como funciona a unidade do Paraná para qualquer fornecedor, cliente e visitantes, por exemplo;

- Controle da estratégia - contém o institucional, mapa estratégico da siderurgia, mapa estratégico CSN Paraná, macro processos, Scorecard do Sistema de Gestão e Meio ambiente, Indicadores de Gestão da Qualidade e Meio Ambiente e relatórios do Cognos;

- Abordagem por processo - é nessa parte que se utilizou o conceito de indicadores de esforço e resultado, como comentado anteriormente. Cada processo possui seu respectivo indicador, os quais estão atribuídos aos seus devidos objetivos estratégicos. 
A fase de execução da reestruturação do modelo de gestão também possibilitou, através do SeSuite, a chamada de outros sistemas especialistas de dentro da própria ferramenta, ou seja, o usuário consegue acessar outro sistema sem sair do SeSuite, pois o sistema é exibido dentro da própria janela do SeSuite e não em outra janela do navegador. Se citar como exemplo o sistema RTPM, o qual contém resultados on-line de indicadores, ao acessá-lo o usuário pode verificar os dados do mês, semana e dia correntes. Dessa maneira, a análise pode ser feita de forma completa, pois o SeSuite contém as informações dos meses passados e o RTPM as informações do mês atual.

Outra possibilidade dessa fase foi o desdobramento dos indicadores em subindicadores. Na sistemática anterior o resultado dos indicadores era divulgado, porém caso fosse necessário alguma análise mais detalhada sobre esse número, era preciso levantar as variáveis que compunham o mesmo. A criação de subindicadores forneceu, ao modelo de gestão da CSN Paraná, maior detalhamento, melhor análise, agilidade na busca do dado e rapidez na tomada de decisão.

\section{CONCLUSÃO}

O desenvolvimento e implantação desse projeto trouxeram ganhos quantitativos e qualitativos para a CSN Paraná, pois grande parte do projeto foi desenvolvido utilizando mão de obra interna.

O valor estimado utilizando horas de consultoria/analistas externos para desenvolver o projeto (com acompanhamento da CSN) seria de $\mathrm{R} \$ 594.323,44$, porém foi desenvolvido por equipe própria da CSN. A CSN Paraná teve que desembolsar R\$ 68.110,00 referente a aquisição do Módulo de Desempenho do SeSuite, customizações no SeSuite para o padrão de trabalho da CSN e gastos com viagens para especificações do Cognos. Então, como economia a CSN obteve um valor total de $R \$ 526.213,44$, conforme detalhamento abaixo:

Tabela 2 - Estimativa de consultoria / analistas externos

\begin{tabular}{|lrr|}
\hline \multicolumn{3}{|c|}{ Estimado utilizando horas de consultoria / analistas externos com participação da CSN } \\
\hline \multicolumn{4}{|c}{ HH } & \multicolumn{1}{c|}{$\mathrm{R} \$ \mathrm{HH}$} & \multicolumn{1}{c|}{ TOTAL } \\
\hline Modulo SeDesempenho & $200 \mathrm{R} \$ 100,00$ & $\mathrm{R} \$ 20.000,00$ \\
Customizações SeSuite desempenho & $500 \mathrm{R} \$ 100,00$ & $\mathrm{R} \$ 50.000,00$ \\
Migração cognos & $2504 \mathrm{R} \$ 149,61$ & $\mathrm{R} \$ 374.623,44$ \\
Configuração sistema gestao qualidade e meio ar & $840 \mathrm{R} \$ 100,00$ & $\mathrm{R} \$ 84.000,00$ \\
Melhorias sistema de gestao e meio ambiente & & \\
Configuração sistema de gestao SST & $438 \mathrm{R} \$ 150,00$ & $\mathrm{R} \$ 65.700,00$ \\
\hline Total serviços estimados & $\mathbf{4 4 8 2}$ & $\mathrm{R} \$ \mathbf{5 9 4 . 3 2 3 , 4 4}$ \\
\hline
\end{tabular}

Tabela 3 - Horas contratadas para implantações

\begin{tabular}{|c|c|c|}
\hline \multicolumn{3}{|c|}{ Horas contratadas para implantações } \\
\hline & RS HH & TOTAL \\
\hline Módulo desempenho & $168 \mathrm{R} \$ 100,00$ & $\mathrm{R} \$ 16.800,00$ \\
\hline Customizações SeSuite desempenho & $500 \mathrm{R} \$ 100,00$ & $\mathrm{R} \$ 50.000,00$ \\
\hline Viagem especificação Cognos & & $\mathrm{R} \$ 1.310,00$ \\
\hline Total Gasto & & $\mathrm{R} \$ \mathbf{6 8 . 1 1 0 , 0 0}$ \\
\hline
\end{tabular}

O projeto também resultou em ganhos significativos de produtividade, como na apuração de dados para consolidação dos resultados, antecipação de reuniões de 
análise crítica, redução de tempo para atualização dos painéis de gestão à vista e acessibilidade a informação. Com isso, foi possível reduzir um efetivo da empresa, o que resultou em um ganho de $\mathrm{R} \$ 107.821,20$.

Veja abaixo o comparativo do cenário anterior com o atual:

Tabela 4 - Comparativo antes $\mathrm{x}$ depois

\begin{tabular}{|c|c|c|}
\hline ITEM & CENÁRIO ANTERIOR & CENÁRIO ATUAL \\
\hline $\begin{array}{l}\text { Antecipação das análises críticas mensais dos } \\
\text { indicadores }\end{array}$ & Geralmente ocorria no dia 14 & Ocorre no dia 7 \\
\hline $\begin{array}{l}\text { Redução do tempo de disponibilização dos } \\
\text { indicadores nos quadros de gestão à vista }\end{array}$ & disponibilizado 3 dias após consolidação & $\begin{array}{c}\text { Disponibilizado em } 1 \text { dia, no máximo, após a } \\
\text { consolidação }\end{array}$ \\
\hline $\begin{array}{l}\text { Análise dos indicadores é registrada no sistema } \\
\text { antes da reunião }\end{array}$ & Não era possível & É possível \\
\hline Descontinuidade do Impromptu pelo fabricante & $\begin{array}{l}\text { Sem possibilidade de crescimento com a } \\
\text { ferramenta devido à descontinuidade pelo } \\
\text { fornecedor } \\
\text { Risco operacional caso o sistema ficasse } \\
\text { indisponível }\end{array}$ & $\begin{array}{c}\text { Sistema implantado com garantia pelo fornecedor } \\
\text { Ajustes feitos pelo próprio usuário }\end{array}$ \\
\hline Impossibilidade de aquisição de novas licenças & Acesso limitado a 4 usuários na CSN Paraná & $\begin{array}{c}\text { Novas licenças, quando necessário, adquiridas } \\
\text { em contrato corporativo (hoje } 54 \text { licenças de } \\
\text { visualização e } 2 \text { de desenvolvedor) }\end{array}$ \\
\hline $\begin{array}{c}\text { Divergência de informações devido a várias } \\
\text { "bases" }\end{array}$ & $\begin{array}{c}\text { Informações desconexas } \\
\text { Regras de negócio existentes nos relatórios não } \\
\text { relatavam mais a realidade } \\
\text { Armazenamento de relatórios em diretórios } \\
\text { pessoais } \\
\text { Dependência de pessoas para obter os números } \\
\text { Números conflitantes }\end{array}$ & $\begin{array}{c}\text { Relatórios padronizados } \\
\text { Regras de negócio adequadas à realidade da } \\
\text { CSN Paraná } \\
\text { Acesso ao sistema para consulta }\end{array}$ \\
\hline Dependência de pessoas para obter informações & Pequenos núcleos de informações nas áreas & Informação disponível a todos \\
\hline $\begin{array}{c}\text { Dificuldade de análise crítica dos indicadores em } \\
\text { reuniões gerenciais }\end{array}$ & $\begin{array}{l}\text { Indicadores em Excel com número fechado } \\
\text { Impossibilidade de análise direta dos números } \\
\text { devido ausência deles no indicador } \\
\text { Caso análise detalhadas fossem necessárias, } \\
\text { havia necessidade de alocação de analista para } \\
\text { levantamento das informações históricas }\end{array}$ & $\begin{array}{l}\text { Desdobramento de todos os indicadores de } \\
\text { desempenho em subindicadores } \\
\text { Independência de todos na analise dos } \\
\text { indicadores de performance na CSN Paraná } \\
\text { Acesso imediato a dados históricos, análises } \\
\text { estatísticas, cruzamento de indicadores, etc. }\end{array}$ \\
\hline $\begin{array}{l}\text { Retrabalho para garantir acuracidade dos } \\
\text { indicadores }\end{array}$ & $\begin{array}{l}\text { Geração de indicadores de forma manual } \\
\text { Erros de digitação, erros no cálculo }\end{array}$ & Indicadores 100 confiáveis \\
\hline Elevado tempo para atualização dos indicadores & $\begin{array}{l}\text { Anteriormente era necessário } 4 \text { dias de um } \\
\text { recurso para consolidação mensal dos } \\
\text { indicadores ( } 32 \mathrm{~h} / \text { mês) }\end{array}$ & $\begin{array}{l}\text { 2h/mês, gerando redução de } 93,75 \% \text { do tempo } \\
\text { (360h no ano) }\end{array}$ \\
\hline
\end{tabular}

Outros resultados significativos foram a proteção dos indicadores e informações, pois agora não estão mais vulneráveis e espalhados em planilhas pelos diretórios de rede, melhoria visual no mapa estratégico e árvore de indicadores, o que facilita a análise durante as reuniões, e o portal para acesso aos outros sistemas especialistas.

Além disso, a estrutura do modelo de sistema de gestão implantando também permitiu uma maior facilidade e agilidade na implantação do projeto do sistema de gestão SST - OHSAS 18001. Algumas sistemáticas dos sistemas de gestão ISO 9001, ISO TS 16949, ISO 14001 foram transferidas e adequadas para o sistema de gestão SST OHSAS 18001, bem como:

- Requisitos legais e outros;

- Objetivos, indicadores, metas e programas;

- Competência, treinamento e conscientização;

- Controle de documentos;

- Preparação e resposta a emergências;

- Monitoramento e medição do desempenho da SST;

- Avaliação do atendimento a requisitos legais e outros;

- Não-conformidades, ação corretiva e preventiva;

- Controle de registros; 
- Auditoria Interna;

- Análise crítica pela direção

Dessa forma a equipe racionalizou recursos como: tempo dispendido na elaboração de documentos, quantidade de documentos, tempo no desenvolvimento do modelo de sistema de medição (indicadores) e comunicação dos resultados.

\section{Agradecimentos}

Agradecemos ao nosso patrocinador Carlos Frederico Rangel Xavier, aos nossos colegas da Diretoria de Informática pelo apoio concebido durante cada fase desse projeto, aos parceiros da Soft Expert e do Grupo GCl por toda atenção e também queremos agradecer a cada funcionário da CSN Paraná, que tenha contribuído diretamente ou indiretamente para o sucesso do grupo como um todo, pois afinal, como diz a nossa Missão, trabalhamos em equipe de forma sustentável para produzir soluções em aço com alta rentabilidade e desempenho diferenciado.

\section{REFERÊNCIAS}

1 Kaplan, R. S.; Norton, D. P. Mapas estratégicos: convertendo ativos intangíveis em resultados tangíveis. Rio de Janeiro: Elsevier, 2004. 